\title{
Neurotrophin signaling pathway in development of AMD-like retinopathy
}

\author{
M.D. Matytsin*, O.S. Kozhevnikova, E.A. Rudnitskaya, D.V. Telegina \\ Institute of Cytology and Genetics SB RAS, Novosibirsk, Russia \\ *e-mail:telegina@bionet.nsc.ru
}

Key words: aging, neurotrophic factors, retinopathy, AMD, NGF, BDNF, OXYS rats

Motivation and Aim: Age-related macular degeneration (AMD) is complex, multifactorial disease that is the main cause of vision loss in people over than 60 years old. As neurotrophins manage neuronal survival, death and synaptic plasticity, we hypothesized that the neurodegenerative changes in the retina during aging and AMD development may be accompanied by alterations in neurotrophin signaling pathway/supplementation, which may be caused by alterations of their level or their bioavailability. To investigate a link between age-related alterations of neurotrophin signaling pathway and progression of AMD we used senescence-accelerated OXYS rats as a suitable model of AMD.

Methods: The RNA-seq data obtained from the retinas of 20 day-, 3-, and 18-month-old OXYS and Wistar (control) rats were used to analyze changes in neurotrophin signaling pathway. Immunohistochemistry (IHC) was used to localize neutrophic factors proBDNF and NGF, and their corresponding receptors $\mathrm{p} 75^{\mathrm{NTR}}$ and TrkA.

Results: Based on comparative analysis of the transcriptome of retina of OXYS and Wistar rats, differently expressed genes from neurotrophin signaling pathway were identified at the preclinical (20 d), the early (3 mo) and the advanced (18 mo) stages of retinopathy development in OXYS rats. These groups were functionally annotated using bioinformatics databases DAVID and KEGG. At the age of $20 \mathrm{~d}$ the expression of nine genes were changed: 6 genes were downregulated (Calm2, Rapla, Rap1b, Rps6ka3, Rps6ka6, Sos2) and 3 genes were upregulated (Atf4, Mapk13, Mapk14). At the age of 3 mo the expression of 7 genes were changed: 6 genes downregulated (Foxo3, Nfkb1, Nfkbia, Pdk1, Rps6ka2, Shc3) and Pik3r2 were upregulated. During the advanced stage of retinopathy (18 mo), the expression of 19 genes was altered: 12 genes were downregulated (Arhgdib, Camk2g, Irak2, Jun, Mapk13, Nfkb1, Nfkbia, Ntf3, Rapla, Raplb, Rps6kal and Ywhah) and 7 genes were upregulated (Mapk12, Nfkbib, Ngfr, Ntrk2, Rapgef1, Rps6ka2 and Sos 1). The products of these genes were associated with the development of the nervous system and signal transduction. According to the IHC, we revealed the increased colocalization of proBDNF with its receptor $\mathrm{p} 75^{\mathrm{NTR}}$, in the retina of OXYS rats at $20 \mathrm{~d}$ and $18 \mathrm{mo}$, indicating shift in neurotrophic balance toward an increase of cell death. Importantly, we found the increased colocalization of NGF and TrkA in 18 mo associated with gliosis of Muller cells.

Conclusion: Considerable alterations of neurotrophin signaling pathway were found at the advanced stage of AMD-like retinopathy development in OXYS rats.

Acknowledgements: The experiments in OXYS and Wistar rats at the age of 3 and 18 mo were supported by the budget project No. 0324-2018-0019, whereas the analysis of 20-day-old rats was supported by the RFBR according to the research project No. 18315-00216. 